\title{
A Novel Low Loss, Highly Birefringent Photonic Crystal Fiber in THz Regime
}

\author{
Hasanuzzaman, G. K. M.; Rana, Sohel; Habib, Selim
}

Published in:

IEEE Photonics Technology Letters

Link to article, DOI:

10.1109/LPT.2016.2517083

Publication date:

2016

Document Version

Peer reviewed version

Link back to DTU Orbit

Citation (APA):

Hasanuzzaman, G. K. M., Rana, S., \& Habib, S. (2016). A Novel Low Loss, Highly Birefringent Photonic Crystal Fiber in THz Regime. IEEE Photonics Technology Letters, 28(8), 899-902.

https://doi.org/10.1109/LPT.2016.2517083

\section{General rights}

Copyright and moral rights for the publications made accessible in the public portal are retained by the authors and/or other copyright owners and it is a condition of accessing publications that users recognise and abide by the legal requirements associated with these rights.

- Users may download and print one copy of any publication from the public portal for the purpose of private study or research.

- You may not further distribute the material or use it for any profit-making activity or commercial gain

- You may freely distribute the URL identifying the publication in the public portal

If you believe that this document breaches copyright please contact us providing details, and we will remove access to the work immediately and investigate your claim. 


\title{
A Novel Low Loss, Highly Birefringent Photonic Crystal Fiber in THz Regime
}

\author{
G.K.M. Hasanuzzaman, Student Member, IEEE, Sohel Rana, Md. Selim Habib, Student Member, \\ IEEE
}

\begin{abstract}
We report on a new kind of dual-hole unit based porous-core hexagonal photonic crystal fiber (H-PCF) for lowloss guidance of $\mathrm{THz}$ radiation. The proposed fiber can offer simultaneously high birefringence and low effective material loss (EML) in the frequency range of $0.5-0.85 \mathrm{THz}$ with single mode operation. An air-hole pair is used inside the core instead of elliptical shaped air-holes to introduce asymmetry for attaining high birefringence; where the birefringence can be enhanced by rotating the dual-hole unit axis of orientation. The proposed $\mathrm{H}$ PCF provides a birefringence of $\sim 0.033$ and an EML of $0.43 \mathrm{~dB} / \mathrm{cm}$ at operating frequency of $0.85 \mathrm{THz}$.
\end{abstract}

Index Terms-Photonic crystal fiber; Terahertz; High birefringence; Effective material loss.

\section{INTRODUCTION}

$\mathrm{R}$ ecently, the limelight has turned towards the latest hot topic terahertz $(\mathrm{THz})$ band ranging from 0.1 to $10 \mathrm{THz}$ due to its enormous applications in the field of sensing [1], imaging [2], security [3], biotechnology [4], spectroscopy [5] and astronomy [6]. Although, there has been a remarkable technological advancement in $\mathrm{THz}$ wave generation and detection techniques, still to date most of the $\mathrm{THz}$ systems that are commercially available are based on free space propagation due to the lack of low loss, flexible and commercially available waveguides [7, 8]. Albeit, dry air exhibits lowest absorption and dispersion than other material in $\mathrm{THz}$ band, free space propagation is not enough convenient due to unavoidable losses introduce during coupling, transporting and managing $\mathrm{THz}$ beam [7-10]. Moreover, waveguides are indispensable in certain cases; when the application point is inaccessible, it is required to interact with the sample strongly and to confine or focus to a smaller spot size [9]. Still, it is a challenge to implement flexible, efficient and low-loss transmission of broadband $\mathrm{THz}$ waves for long length delivery. In order to lower the propagation loss of a $\mathrm{THz}$ waveguides, an effective approach is to confine large part of radiation in air while propagate small portion to material $[11,12]$.

Different types of $\mathrm{THz}$ waveguides have been addressed both theoretically and experimentally based on metal wires

The authors are with the Department of Electrical and Electronic Engineering, Rajshahi University of Engineering and Technology, Rajshahi6204, Bangladesh (g.kibria82@yahoo.com, selim041073@yahoo.com).

Md. Selim Habib is also with the DTU Fotonik, Department of Photonics Engineering, Technical University of Denmark, DK-2800 Kgs. Lyngby, Denmark. (e-mail: seha@fotonik.dtu.dk). and dielectric sub-wavelength fibers. These include dielectric metal-coated tubes, metallic wires, Bragg fibers, and alldielectric sub-wavelength polymer fibers [13-18]. Though sub-wavelength fibers and metallic wires exhibit low absorption loss in $\mathrm{THz}$ range, most of the field propagates outside the waveguide core, thus resulting in strong coupling to the environment. On the contrary solid core of the conventional photonic crystal fibers (PCFs) shows a high material absorption loss [18], which is almost equal to the bulk material absorption loss. Recently the spotlight has turned to porous-core photonic crystal fiber, which offers relatively lower absorption loss than a PCF with porous cladding or porous-core fiber with air cladding $[7,8,11,12]$. Along with the low loss property, high birefringence is also desired in numerous applications like sensing, communications, and polarized terahertz filters $[10,19]$. Two popular methods are mainly used to achieve high birefringence in porous fibers just like as conventional PCFs. One is to break the symmetry of the fiber cladding and another is to introduce asymmetry to the fiber core [10]. For the first time, Atakaramians et al. [20] designed and fabricated Polymethyl-methacrylate (PMMA) based rectangular porous fibers to achieve low loss and high birefringence. The reported value of birefringence is 0.012 at $f=0.65 \mathrm{THz}$. A birefringence level of $10^{-2}$ has been achieved by Chen et al. [21] by means of a squeezed lattice ellipticalhole. As elliptical air-holes introduce additional fabrication challenge and reduce coupling efficiency, this kind of porous fiber is inconvenient for practical application [10]. A Topas based porous fiber with a hexagonal array of sub-wavelength elliptical holes have been identified as a means of achieving low loss, high birefringence and single mode $\mathrm{THz}$ guidance by Chen et al. [10]. Numerical investigations in [10] show that birefringence can be enhanced by rotating the major axis direction of the elliptical air-holes and there exists an optimal rotating angle at $30^{\circ}$. At this optimal angle a birefringence as high as 0.0445 can be obtained at a frequency range from 0.73 to $1.22 \mathrm{THz}$. However, this kind of elliptical air-hole based fiber is difficult to fabricate and a certain amount of power is propagating outside the fiber structure due to air cladding.

In this paper, we have demonstrated a novel and relatively simple technique of attaining high birefringence and low loss simultaneously without using elliptical air-holes as most of literature used to achieve high birefringence properties. The proposed PCF incorporates both a porous-core and also a porous-cladding of hexagonal array structure. Instead of inserting single elliptical air-hole we introduced two circular 
air-holes of same size inside the core, thereby the proposed fiber provides important manufacturing benefits and we define the air-hole pair as dual-hole unit. The axis of orientation of the dual-hole unit is rotated to obtain high birefringence. Numerical simulations show that rotation angle of $30^{\circ}$ exhibits optimal results at which the fiber exhibits high birefringence and considerable low absorption loss with single mode operation.

\section{GEOMETRY OF THE PROPOSED DESIGN}

The proposed H-PCF is based on a dual-hole unit where the two small air-holes in one unit can be effectively viewed as an elliptical air-hole as shown in Fig.1. Hexagonal lattice of three air-hole rings have been used in both core and cladding to reduce complexity. In the cladding region, the spacing between two adjacent air-holes on two adjacent rings is denoted as $\Lambda_{s}$. $D_{\text {core }}$ denotes the core diameter, where diameter of the core and cladding air-holes are labeled as $d_{c}$ and $d$ respectively (see Fig.1). $\Lambda_{c}$ stands for unit to unit distance whereas $\mathrm{H}$ is the centre to centre distance between two airholes of a dual-hole unit. The air-hole diameter to pitch ratio in the cladding $d / \Lambda_{s}$ is kept 0.90 throughout the simulation. Furthermore, the orientation of air-holes inside the core at different rotation angle of the proposed porous-core hexagonal PCF is depicted in Fig.2. The size and the rotation angle of the air-holes in the core have a large contribution for attaining high birefringence and low absorption loss. The parameters used for the simulations are $D_{\text {core }}=350 \mu \mathrm{m}, d=286 \mu \mathrm{m}, \Lambda_{s}=$ $318 \mu \mathrm{m}, \Lambda_{c}=49.3 \mu \mathrm{m}, d_{c}=22.68 \mu \mathrm{m}$ and $\mathrm{H}=24.5 \mu \mathrm{m}$. We used a cyclic-olefin copolymer (COC) with the trade name TOPAS as background material. The selection based on of its unique and beneficial properties which include low bulk material loss of $0.2 \mathrm{~cm}^{-1}$ [8], constant refractive index $n=1.5258$ in the $\mathrm{THz}$ spectrum which is beneficial for near zero dispersion [22], humidity insensitivity [12], chemically inertness with special bio-sensing properties and does not absorb water [12].
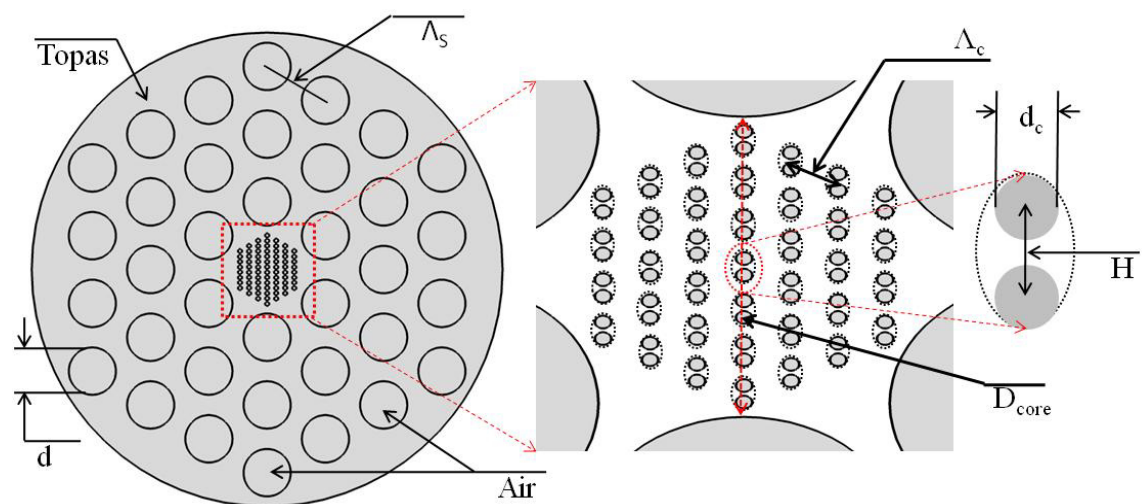

Fig. 1. Cross section of proposed hexagonal structure with three air-hole rings in the cladding and three air-hole rings in the core at the rotation angle $90^{0}$.

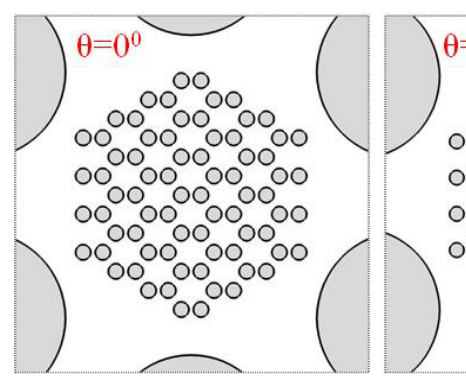

(a)

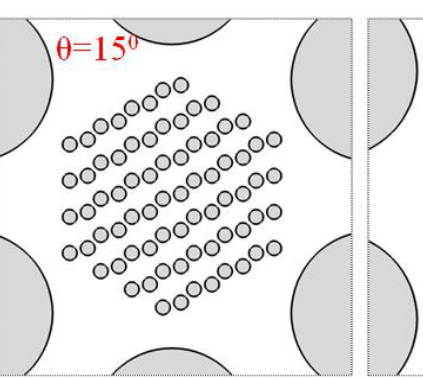

(b)

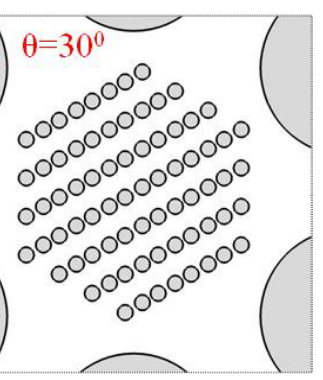

(c)

Fig. 2. Anti-clockwise rotation of the axis of orientation of dual-hole unit (a) $0^{\circ}$ (b) $15^{\circ}$, and (c) $30^{\circ}$.

\section{NUMERICAL RESULTS AND DISCUSSION}

In this paper, birefringence is obtained by introducing asymmetry inside the core. The axis direction of dual-hole unit might play an important role in introducing asymmetry [10] and hence the birefringence might be varied when one rotates the axis direction. Birefringence $B$ can be obtained by the following equation [12]

$$
B=\left|n_{x}-n_{y}\right|
$$

where $B$ is the birefringence, $n_{x}$ and $n_{y}$ are the refractive indices of $x^{-}$and $y-$ polarization respectively. The birefringence as a function of frequency at different rotation angle is shown in Fig. 3. It is seen from Fig. 3 that the birefringence increases gradually with the increase of rotation angle. When the rotation angle becomes $30^{\circ}$, the birefringence reaches the zenith point. Further increment of rotation angle, there is a decrement of birefringence. The structure of the fiber and the layout of air-holes in the core explain the angledependent variation. For example, when the angle is rotated to $30^{\circ}$, there exists the most difference of the porosity between the two orthogonal polarizations directions corresponds to the maximal birefringence. It should be noted that, when being rotated to $60^{\circ}$, the geometric configuration of the fiber is 
identical to that of unrotated state owing to the six-fold symmetry of the hexagonal porous fiber.

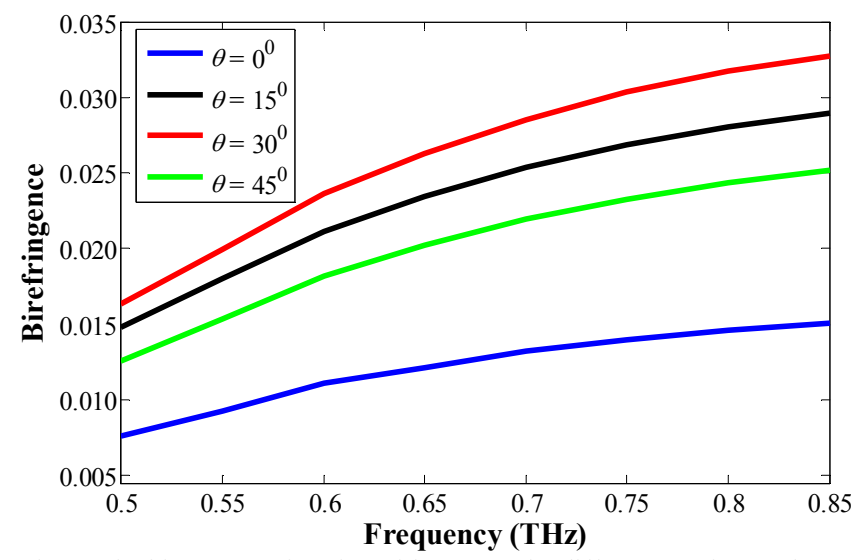

Fig. 3. Birefringence as function of frequency for different rotation angle.

All simulations in our design are performed under the single mode condition which is determined by a parameter named $\mathrm{V}$ parameter expressed by [10]

$$
V=\frac{2 \pi r f}{c} \sqrt{n_{c o}^{2}-n^{2} c l} \leq 2.405
$$

where $r$ is the radius of the fiber core, $f$ is the frequency, $c$ is the velocity of light in vacuum and $n_{\mathrm{co}}$ and $n_{\mathrm{cl}}$ are the effective indices of the fiber core and cladding respectively. As the cladding structure is mostly holey, $n_{c l}$ can be approximated as 1. V-parameter versus as a function of frequency is depicted in Fig. 4 which shows that the single mode cutoff frequency is around $0.85 \mathrm{THz}$. Although single mode exists at a frequency lower than $0.5 \mathrm{THz}$, due to the relatively low value of birefringence in this band, simulations are performed over 0.5 to $0.85 \mathrm{THz}$ frequency range.

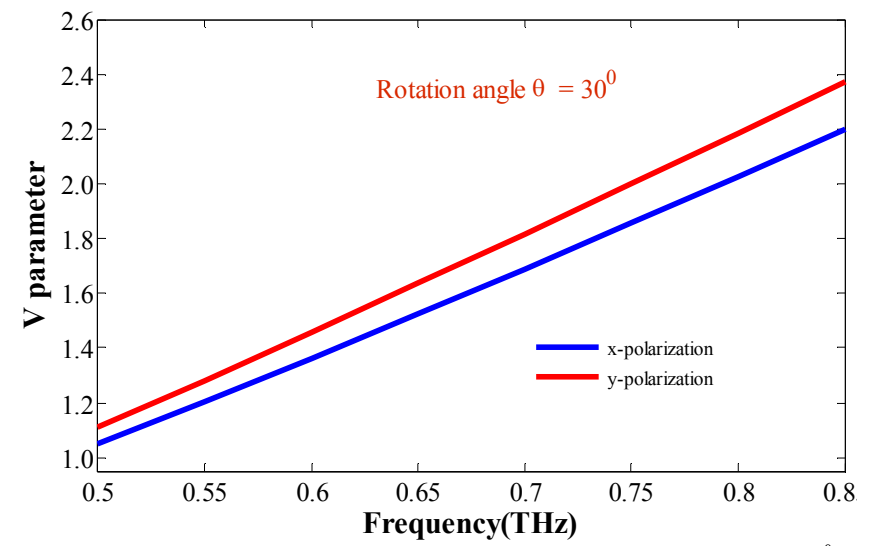

Fig. 4. V-parameter as a function of frequency at optimal rotation angle $30^{\circ}$ for $\mathrm{x}$ - and $\mathrm{y}$ - polarization.

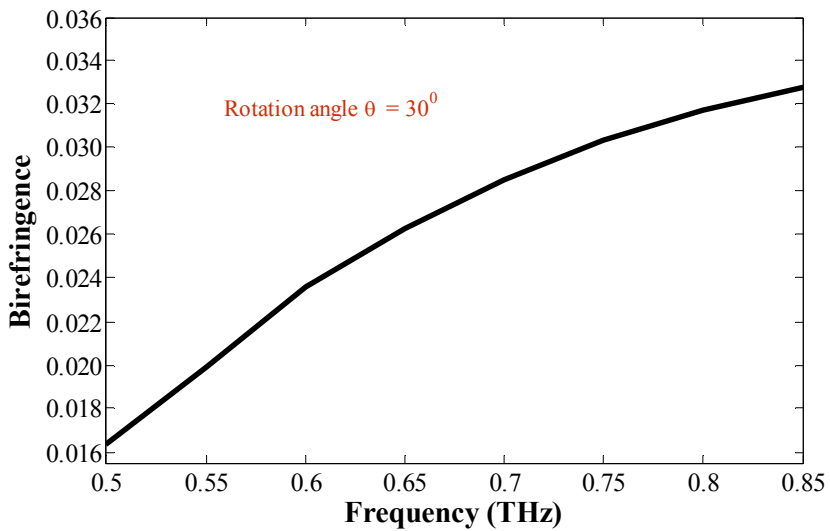

Fig. 5. Frequency dependence of birefringence at optimal rotation angle.

Figure 5 depicts the birefringence as function of frequency. The birefringence as high as $\sim 0.033$ is achieved at frequency $0.85 \mathrm{THz}$ which is much higher compared with those reported on Refs. [20-21]. Power flow distribution in the $z$-direction for two orthogonal polarization modes at different rotation angle is shown in Fig. 6 at $0.85 \mathrm{THz}$. The figures indicate that the mode power is well confined in the fiber core.

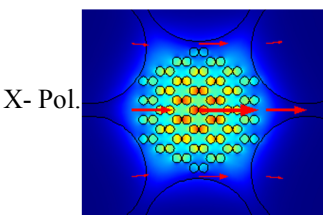

(a)

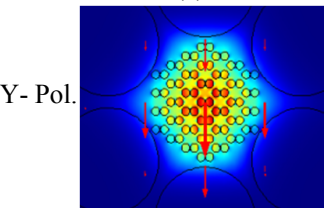

(d)

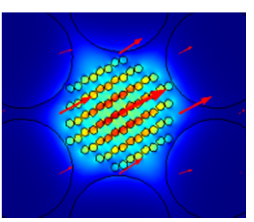

(b)

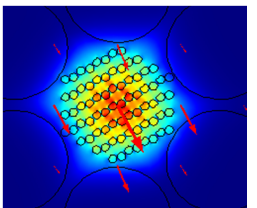

(e)

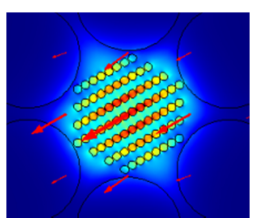

(c)

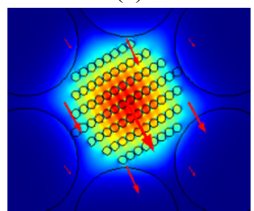

(f)
Fig. 6. Power flow distributions in the $\mathrm{z}$ direction for $\mathrm{x}$ - and $\mathrm{y}$ - polarization. Power flow distributions are shown for fundamental modes at 0.85 . THz. (a) (b) and (c) for x-polarization at $0^{\circ}, 15^{\circ}$, and $30^{\circ}$ rotation angles respectively. (d), (e) and (f) for y-polarization at $0^{\circ}, 15^{\circ}$, and $30^{\circ}$ rotation angle respectively.

Effective material loss (EML) or absorption loss $\left(\alpha_{e f f}\right)$ is used to investigate the loss property of the PCFs for terahertz frequencies. Effective material loss can be calculated by the following equation [22]

$$
\alpha_{\text {eff }}=\frac{1}{2}\left(\frac{\varepsilon_{0}}{\mu_{0}}\right)^{1 / 2} \frac{\int_{A_{\text {mat }}} n_{\text {mat }} \alpha_{\text {mat }}|E|^{2} d A}{\left|\int_{\text {all }} S_{z} d A\right|}
$$

where $\varepsilon_{0}$ and $\mu_{\mathrm{o}}$ are the permittivity and the permeability respectively in the vacuum, $\alpha_{\text {mat }}$ is the material absorption loss, $n_{\text {mat }}$ is the refractive index of the background material. It should be pointed out that, in Eq. (3), the integration in the numerator is only performed over the solid material region $\left(A_{\text {mat }}\right)$, whereas denominator is performed over all regions. 


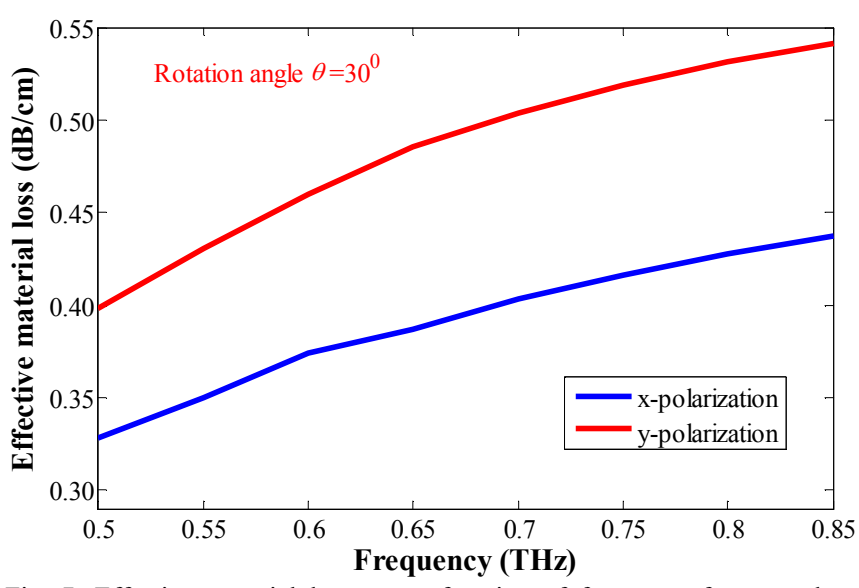

Fig. 7. Effective material loss as a function of frequency for $\mathrm{x}$ - and $\mathrm{y}$ polarization at optimal rotation angle $\theta=30^{\circ}$.

Figure 7 depicts the effective material loss as a function of frequency at $\theta=30^{\circ}$. It can be seen that the EMLs of $\mathrm{x}$ - and $\mathrm{y}$ polarization modes are $0.43 \mathrm{~dB} / \mathrm{cm}$ and $0.54 \mathrm{~dB} / \mathrm{cm}$, respectively at $0.85 \mathrm{THz}$. Therefore, the loss of $\mathrm{x}$ - polarization mode is decreased to $50 \%$ of its bulk material loss which is good enough for practical applications. Moreover the EML of $\mathrm{x}$ - polarization is smaller than that of y-polarization. More electromagnetic fields exist in Topas for $y$ - polarization since $\mathrm{y}$ - polarization's refractive index is higher than x-polarization. The variation of refractive index as a function of frequency is demonstrated in Fig. 8.

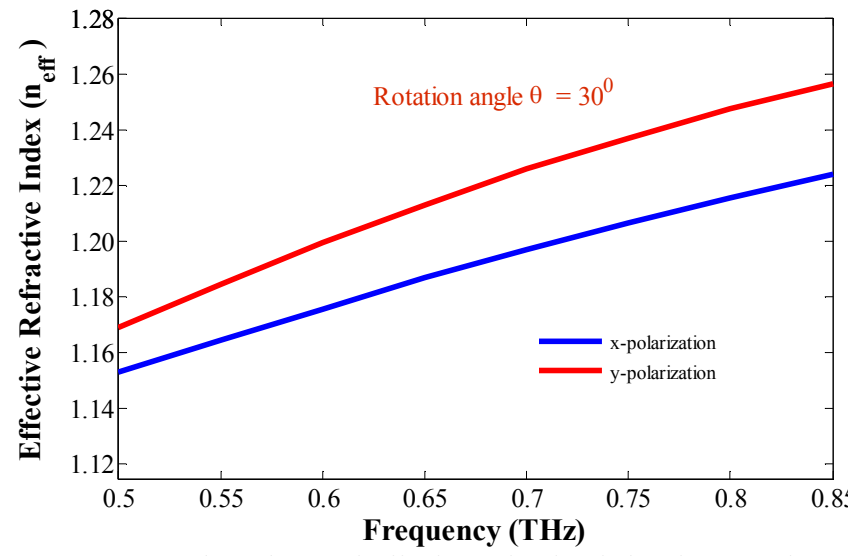

Fig. 8. Frequency dependence of effective refractive index for $\mathrm{x}$ - and $\mathrm{y}$ polarization at optimal rotation angle $\theta=30^{\circ}$.

Another kind of loss mechanism usually occurred in photonic crystal fiber is known as confinement loss usually occurs due to the finite extent of the periodic cladding and is obtained from the imaginary part of the complex refractive index, $n_{\text {eff }}$ is given by

$$
\alpha_{C L}=\left(\frac{8.686}{100}\right)\left(\frac{2 \pi f}{c}\right) \operatorname{Im}\left(n_{e f f}\right) \quad \mathrm{dB} / \mathrm{cm}
$$

where $f$ the frequency of the light is, $c$ is the speed of the light in vacuum and $\operatorname{Im}\left(n_{\text {eff }}\right)$ is the imaginary part of the refractive index of the guided mode. Confinement loss as a function of frequency at optimum rotation angle $\theta=30^{\circ}$ is shown in Fig. 9. It can be observed that calculated value of confinement loss is far below the level of effective material loss and hence can be neglected.

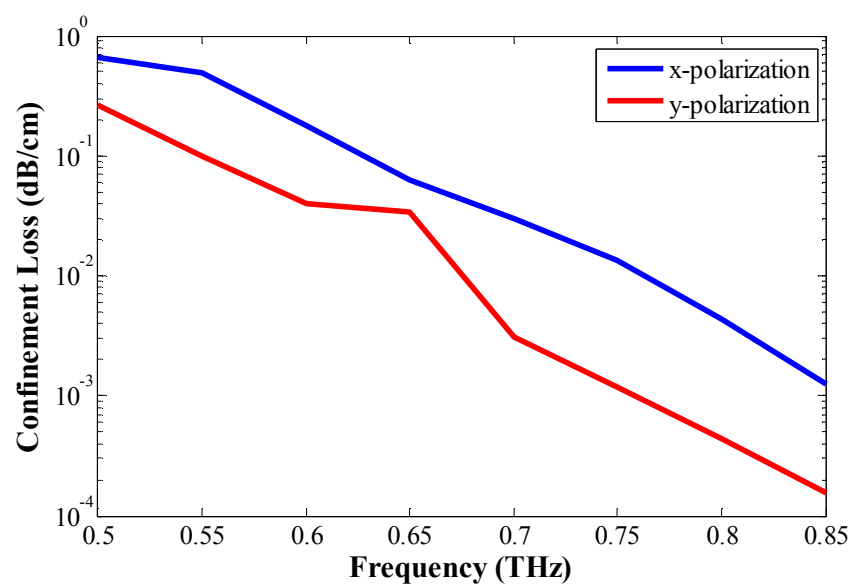

Fig. 9. Confinement loss as function of frequency for $\mathrm{x}$ - and $\mathrm{y}$-polarization at optimal rotation angle $\theta=30^{\circ}$.

Another important property that represents the power flow distribution of different regions named fraction of mode power can be estimated by the following equation [10]

$$
\eta^{\prime}=\frac{\int_{X} S_{Z} d A}{\int_{\text {all }} S_{Z} d A}
$$

$X$ may be one of three regions among air-holes in core, background material and air cladding. Figure 10 shows the fraction of power in different regions as functions of frequency for two orthogonal polarization modes at optimum rotation angle of $30^{\circ}$. It is seen from Fig. 10 that about $32.5 \%$ of total power confines in core air-holes for x-polarization and $28 \%$ for y-polarization at operating frequency $f=0.85 \mathrm{THz}$. One of the most noticeable points is that in this case all the power is confined inside the waveguide whereas; a fraction of light energy is transported outside the fiber for porous fiber with air cladding [10].

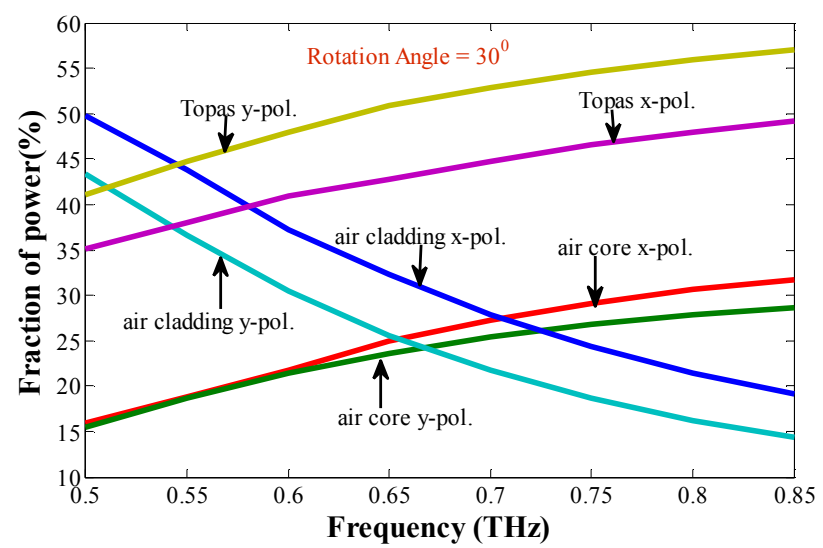

Fig. 10. Fraction of power localized in air-core, air cladding and Topas for both orthogonal polarization modes for $30^{\circ}$ rotation angle within the single mode operating region.

Finally, we would like to address the fabrication feasibility of the proposed design. At present PCFs fabrication is no 
longer a changeling issue due to the intense technological advancement. Especially PCFs for terahertz frequency regime application with 20 to $40 \mu \mathrm{m}$ inner pitch dimension are easier to fabricate than those PCFs operating at optical frequencies with sub-micrometer pitch [7]. Meanwhile, PCFs operating at terahertz frequency range have already been fabricated [12]. The design proposed here is relatively simper and includes only circular shaped air-holes in hexagonal lattice. Therefore, the proposed structure can be fabricated by using most versatile stack and draw technology.

\section{CONCLUSION}

We have designed and analyzed a single mode THz-PCF with high birefringence and low losses. To attain high birefringence, a dual-hole unit has been used instead of single elliptical air-hole which might offer great fabrication benefits. Numerical investigation showed that birefringence can be enhanced by rotating the dual-hole unit axis of orientation and there exist an optimum rotation angle at $30^{\circ}$. Birefringence of $\sim 0.033$ and EML of $0.43 \mathrm{~dB} / \mathrm{cm}$ was obtained at frequency $0.85 \mathrm{THz}$ with very low confinement loss. The proposed THzPCF can be beneficial for polarization maintaining $\mathrm{THz}$ wave guidance with potential applications like polarized $\mathrm{THz}$ filters and sensors.

\section{References}

[1] R.H. Jacobsen, D.M. Mittleman, and M.C. Nuss, "Chemica recognition of gases and gas mixtures with terahertz waves," Opt. Lett., vol. 21, no. 24, pp. 2011-2013, 1996.

[2] Q. Chen, Z. Jiang, G.X. Xu, and X.C. Zhang, "Near-field terahertz imaging with a dynamic aperture," Opt. Lett. vol. 25 no. 15, pp. 1122-1124, 2000.

[3] A. Hassani, A. Dupuis, and M. Skorobogatiny, "Porous polymer fibers for low-loss Terahertz guiding," Opt. Express, vol. 16, no. 9 , pp. $6340-6351,2008$.

[4] N. Laman, S.S. Harsha, D. Grischkowsky, and J.S. Melinger, "7 $\mathrm{GHz}$ resolution waveguide $\mathrm{THz}$ spectroscopy of explosives related solids showing new features," Opt. Express, vol. 16, no. 6, pp. 4094-4105, 2008.

[5] Y.F. He, P.I. Ku, J.R. Knab, J.Y. Chen, and A.G. Markelz "Protein Dynamical Transition Does Not Require Protein Structure," Phys. Rev. Lett. vol.101, no.17 pp. 178103-178106, Oct.2008.

[6] J. Zhang and D. Grischkowsky, "Waveguide terahertz timedomain spectroscopy of nanometer water layers," Opt. Lett. vol. 29 , no. 14 pp. 1617-1619, 2004.
[7] M. Uthman, B. M. A. Rahman, N. Kejalakshmy, A. Agrawal, and K. T. V. Grattan, "Design and characterization of low-loss porouscore photonic crystal fiber," IEEE photon. J., vol. 4, no. 6, pp. 2315-2325, Dec. 2012.

[8] S. F. Kaijage, Z. Ouyang, and X. Jin, "Porous-Core Photonic Crystal Fiber for Low Loss Terahertz Wave Guiding," IEEE Photon. Technol. Lett., vol. 25, no. 15, pp. 1454-1457, Aug. 2013.

[9] S.Atakaramians, S.Afshar, M.Nagel, H.K.Rasmussen, O.Bang, T. M.Monro, and D. Abbott, "Direct probing of evanescent field for characterization of porous terahertz fibers," Appl. Phys. Lett. vol. 98, pp. 121104-1,121104-3, 2011.

[10] N. Chen, J. Liang, and L. Ren, "High birefrience, low-loss porous fiber for single-mode terahertz-wave guidance," J. Appl. Opt., vol. 52, no. 21, pp. 5297-5302, Jul. 2013.

[11] K. Nielsen, H. K. Rasmussen, P. U. Jepsen, and O. Bang, "Porouscore honeycomb bandgap THz fiber," Opt. Lett., vol. 36, no. 5, pp. 666-668, Mar. 2011.

[12] H. Bao, K. Nielsen, H. K. Rasmussen, P. U. Jepsen, and O. Bang, "Fabrication and characterization of porous-core honeycomb bandgap THz fibers," Opt. Express, vol. 20, no. 28, pp. 2950729517, Dec.2012.

[13] B. Bowden, J. A. Harrington, and O. Mitrofanov, "Silver/polystyrene coated hollow glass waveguides for the transmission of terahertz radiation," Opt. Lett., vol.32, no. 20, pp. 2945-2947, 2007.

[14] K. Wang and D. M. Mittleman, "Metal wires for terahertz wave guiding," Nature, vol. 432, pp. 376-379, Sept. 2004.

[15] T. Jeon, J. Zhang, and D. Grischkowsky, "THz Sommerfeld wave propagation on a single metal wire," Appl. Phys. Lett., vol. 86, pp. 161904-1-161904-3, 2005.

[16] A. Dupuis, K. Stoeffler, B. Ung, C. Dubois, and M. Skorobogatiy, "Transmission measurements of hollow-core THz Bragg fibers, " $J$. Opt. Soc. Amer. B, vol. 28, no.4, pp. 896-907, Apr. 2011.

[17] L.-J. Chen, H.-W. Chen, T.-F. Kao, J.-Y. Lu, and C.-K. Sun, "Lowloss subwavelength plastic fiber for terahertz waveguiding," Opt. Lett., vol. 31, no. 3, pp. 308-310, 2006.

[18] H. Han, H. Park, M. Cho, and J. Kim, "Terahertz pulse propagationin a plastic photonic crystal fiber," Appl. Phys. Lett., vol. 80, no. 15, pp. 2634-2636, 2002.

[19] Y. Guo-Bing, L. Shu-Guang, W. Xiao-Yan, and L. Shuo "High birefringence, low loss terahertz photonic crystal fibres with zero dispersion at 0.3 THz," Chin. Phys. B, vol.20, no.9, 090701-1$090701-4,2011$.

[20] S. Atakaramians, S. Afshar V, H. Ebendorff-Heidepriem, M. Nagel, B. M. Fischer, D.Abbott, and T. M. Monro, "THz porous fibers: design, fabrication and experimental characterization," Opt. Exp., vol. 17, no. 16, pp. 14053-14062, Jul. 2009.

[21] H. B. Chen, D. R. Chen, and Z. Hong, "Squeezed lattice ellipticalhole terahertz fiber with high birefringence," Appl. Opt. vol. 48, no. 20, pp. 3943-3947, 2009.

[22] J. Liang, L. Ren, N. Chen, and C. Zhou, "Broadband, low-loss, dispersion flattened porous-core photonic bandgap fiber for terahertz (THz)-wave propagation," J. Opt. Commun., vol. 295, pp. 257-261, Jan. 2013. 